\title{
Isolating Vector-insert Junctions from Yeast Artificial Chromosomes
}

\author{
Gary A. Silverman
}

Department of Pediatrics, Harvard Medical School, Boston, Massachusetts 02115 USA

he physical mapping of complex genomes can be achieved by assembling contigs of yeast artificial chromosomes (YACs). In the early stages of map construction, randomly generated markers can be used to screen YAC libraries, order the clones, and assemble the contigs. However, as the maps increase in size, this process becomes less efficient, as randomly generated markers are less likely to add new clones to the ends of the contigs. This problem can be overcome by a more directed approach such as the generation of markers from the ends of YAC inserts. The purpose of this report is to consider the practical aspects of the methods used to isolate vectorinsert junctions from YAC clones. Although many techniques are available, the high throughput and success rate of PCR-based strategies may prove to be the most efficient means for closing the gaps between contigs and completing the physical maps of complex genomes.

The advent of yeast artificial chromosome (YAC) cloning systems has facilitated greatly the mapping, cloning, and functional analysis of complex genomes (as reviewed). ${ }^{(1-3)}$ YACs spanning the euchromatic segments of the human $\mathrm{Y}^{(4)}$ and $21^{(5)}$ and extensive portions of the Caenorhabditis elegans ${ }^{(6)}$ and Drosophila melanogaster $^{(7)}$ genomes attest to their importance in genome mapping. The positional cloning of disease-associated genes such as the neurofibromatosis type $1,{ }^{(8)}$ adenomatous polyposis coli (APC),${ }^{(9,10)}$ and the Huntington's disease genes $^{(11,12)}$ also relied on cloning and mapping with YACs.

The physical map of a genomic region can be deduced by the assembly of contiguous clones (contigs). Contigs are a collection of cloned DNA segments with individual members that share overlap with at least one other member of the set. ${ }^{(13)}$ By analyzing partial or complete restriction fragment patterns (fingerprints) of individual $\lambda$ phage or cosmid clones, contigs spanning extensive portions of the Escherichia coli, ${ }^{(14)} \mathrm{C}$. elegans, ${ }^{(15)}$ and Saccharomyces cerevisiae $e^{(13,16)}$ genomes have been constructed. Contig construction using YACs has proved more arduous. The fingerprinting of YACs by restriction mapping, although feasible, ${ }^{(17,18)}$ is confounded by the large genomic insert, repetitive sequences, multiple YACs in a single cell, and chimeric fragments. As an alternative to fingerprinting methods, Green and Olson proposed the concept of sequence tagged site (STS)-content mapping. ${ }^{(19)}$ STSs are small, singlecopy fragments in the genome that can be recovered or detected by unique PCR primers. ${ }^{(20)}$ Conceivably, YAC contigs spanning entire chromosomes could be assembled by using STS-specific PCR assays to screen libraries and detect overlapping clones. This concept was supported by the use of 16 STSs to align 30 YACs into a $1.5-\mathrm{Mb}$ contig spanning the cystic fibrosis gene region. ${ }^{(19)}$ On a larger scale, the generation of additional STSs should permit complete mapping of an entire genome. In the early stages of map construction, the random generation of STSs may prove to be the most efficient method to initiate contig assembly. ${ }^{(21-23)}$ However, if the probability of isolating a random STS is predicted by the Poisson distribution, then the efficiency of map construction will diminish greatly as the contigs expand to cover $60-80 \%$ of the genome. ${ }^{(24)}$ Specifically, calculations based on the Poisson distribution predict that $60 \%$ of the total effort would be required to complete the final $13 \%$ of the map if a random approach is used exclusively. Palazzolo et al. ${ }^{(24)}$ present computer simulations of contig building on the basis of STS generation by random, single-end, clone-limited (isolation of an STS from one end of a clone), and double-end, clone-limited strategies (isolation of STSs from both ends of a clone). Clearly, the generation of STSs via the double-end, clone-limited strategy, especially in the latter stages of map construction, is the most efficient method for completing contig assembly. ${ }^{(24)}$ In practical terms, however, the benefits of contig construction by a directed approach can be offset if methods for isolating STSs from the ends of the clones are inefficient or labor intensive relative to those used for generating random STSs.

The need to develop methods that easily and reliably yield terminal DNA sequences from the genomic insert was apparent early in the evolution of YAC cloning. This was prompted by the simple notion that the termini from YAC inserts will maximize a chromosomal walk by detecting clones in the library with the least amount of overlap. The purpose of this report is to review the utility of the techniques used to isolate YAC vector-insert junctions. The plethora of available techniques suggests that no preferred method has emerged. However, PCR-based techniques, rather than conventional plasmid or $\lambda$ phage subcloning, have experienced the most widespread use (Table 1). All of the techniques provide the means to isolate STSs that can be used to build contigs and fill gaps. In addition, these end fragments can be used to assess the extent of overlap with other clones, determine telomeric-centromeric orientation of YACs, increase marker density for pulsed-field gel electrophoresis (PFGE) mapping, generate new restriction-fragment length polymorphisms, and identify chimeric YAC clones.

The techniques described in this re- 
TABLE 1 Methods for Isolating YAC Vector-insert Junctions

\author{
Plasmid rescue \\ Plasmid rescue after vector arm \\ modifications, in vivo \\ Conventional subcloning \\ $\lambda$ phage \\ plasmids \\ Genomic sequencing \\ PCR \\ Alu-vector \\ anchor-vector \\ inverse
}

port have been successful in isolating vector-insert junctions from the left or right arms of the pYAC series of vectors. ${ }^{(25)}$ For reference, the left arms (LA) of pYAC vectors contain the CEN4, ARS1, and TRP1 sequences, whereas the right arm (RA) contains the URA3 gene. With some modifications, these methods will prove useful in isolating terminal fragments from DNA propagated in other cloning systems (e.g., Pls, BACs, PACs, and cosmids).

\section{PLASMID RESCUE}

The isolation of a YAC vector-insert junction by plasmid rescue was described by Burke et al. ${ }^{(25)}$ YAC DNA is digested with a restriction enzyme that generates a telomere-free junction fragment. The fragments are self-ligated, and the resultant plasmids are used to transform $E$. coli. The advantages of plasmid rescue are its technical simplicity and the ability to obtain fragments as large as $20 \mathrm{~kb}$. These fragments can be used as fluorescence in situ hybridization (FISH) probes, and their larger size also increases the likelihood of obtaining unique sequence information. Unfortunately, the original design of the pYAC series of vectors placed a ColE1 origin of replication and ampicillin resistance gene only on the LA. Furthermore, the limited number of available restriction sites in the vector arm (Xhol, Ndel) limits the usefulness of this technique to isolating those genomic inserts that have either a XholSalI or NdeI rare-cutting restriction site within $\sim 20 \mathrm{~kb}$ of the LA cloning site.

Although the RA does not contain a ColE1 origin of replication or an antibiotic resistance gene, vector-insert junctions can be rescued indirectly. YAC DNA, digested with an enzyme that yields vector-insert fragment containing the URA3 gene, is subcloned into a plasmid vector. The appropriate clone is isolated by URA3 complementation of $E$. coli containing the pyrF mutation. ${ }^{(26)}$

Newer YAC vectors such as pYACneo, ${ }^{(27)}$ pJS97/98, ${ }^{(28)}$ and the pGS966999 series $^{(29)}$ permit plasmid rescue from both sides of the cloning site. They contain ColE1 origins, antibiotic resistance genes, and several common restriction sites in both vector arms. As an added feature, some of these vectors contain T3 or $\mathrm{T} 7$ bacteriophage RNA polymerase promoters that permit riboprobe synthesis from either side of the cloning site.

The widely available Washington University, ${ }^{(30)} \mathrm{CEPH},{ }^{(31)} \mathrm{ICI}^{(32)}$ and $\mathrm{ICRF}^{(33)}$ human genomic YAC libraries were constructed using the pYAC4 vector. To facilitate plasmid rescue directly from these clones, Hermanson et al. ${ }^{(34)}$ developed a set of integrating plasmids to retrofit the pYAC4 vector arms with elements present in the newer vectors: dual ColE1 origins of replication, antibiotic resistance genes, and clusters of restriction sites (Fig. 1). These plasmids, pICL (integrating into CEN side with LYS2) and pLUS (LYS2 integration into the
URA3 side) are linearized by endonuclease digestion and are used to transform yeast via a lithium acetate procedure. Transformants are selected for lysine prototrophy. pICL and pLUS integrate by homologous recombination into the ampicillin resistance gene of the LA and a segment of pBR322-related sequence in the RA, respectively. Integration site-specific PCR assays can be used to confirm that pICL and pLUS are inserted appropriately into the vector arms. By linearizing the plasmids before transformation, $70-100 \%$ of the lysine prototrophs contain integrating vectors at the appropriate pYAC4 site. As expected, transformation with nonlinearized plasmids dramatically reduces the percentage of Lys ${ }^{+}$cells with correct insertions. Plasmid rescue of the YAC vector-insert junctions is achieved by digesting miniprep DNA of the Lys ${ }^{+}$ transformants with a restriction enzyme that cleaves in the polylinker, ligating the restriction fragments, transforming E. coli and selecting for ampicillin (pICL) or kanamycin resistance (pLUS) (Fig. 1). Over 50 end fragments, up to $20 \mathrm{~kb}$ in size, were rescued using this technique.

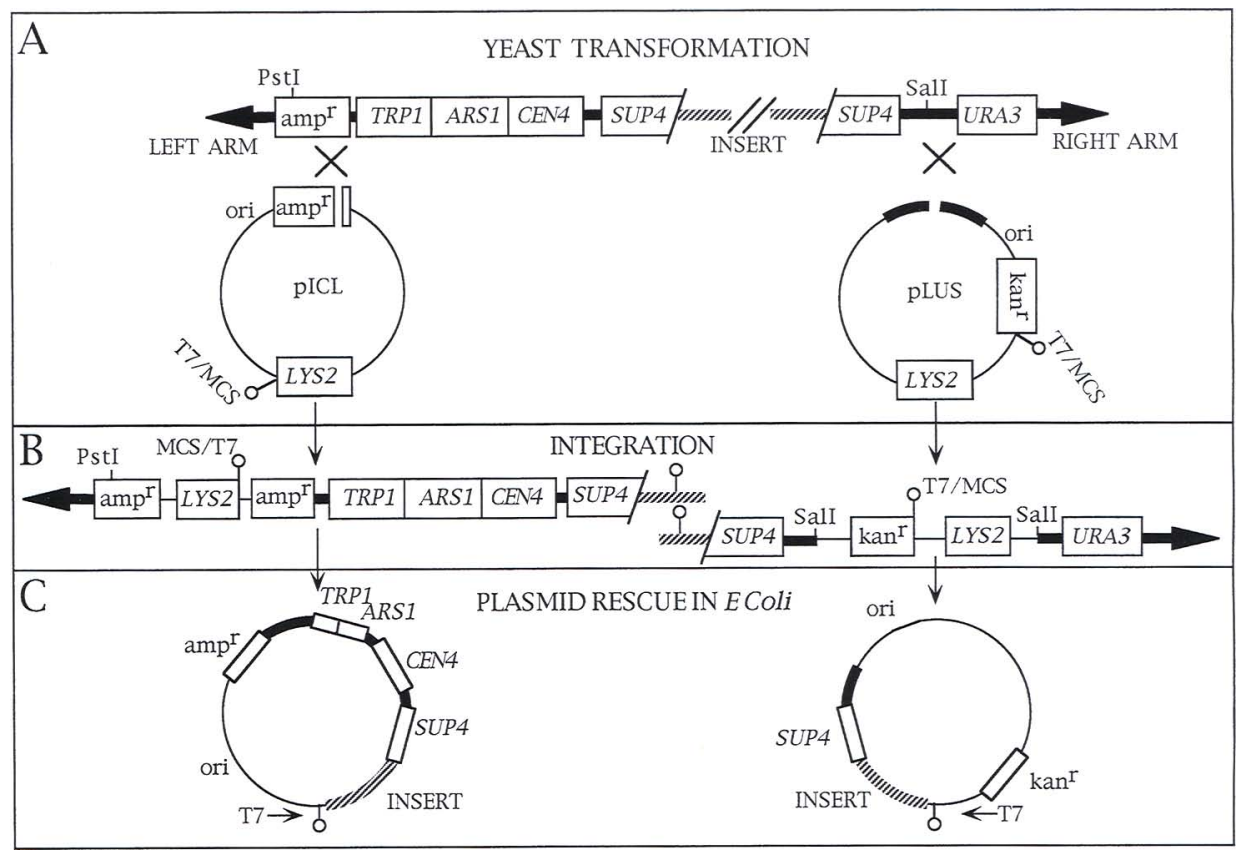

FIGURE 1 Plasmid rescue using integrating vectors. (A) Yeast are transformed with the integrating plasmids pICL or pLUS. These vectors contain a multiple cloning site (MCS), a yeast selectable marker (LYS2), and a segment that is homologous with sequences in either the left or right YAC vector arm. Integrating vectors are linearized before transformation to enhance homologous recombination. (B) Integration of pICL and pLUS into the left and right vector arms, respectively. (C) Plasmid rescue is achieved by digesting YAC DNA with a restriction enzyme that cleaves in the MCS and at an unknown position in the genomic insert $(O)$. The DNA is self-ligated and used to transform E. coli. Adapted, with permission, from Hermanson et al. ${ }^{(34)}$ 
The major disadvantage of this procedure may be the expenditure of time and effort necessary to complete this multistep cloning process. However, the ability to isolate many large-size end fragments may justify this investment for some positional cloning experiments.

\section{CONVENTIONAL SUBCLONING}

Sublibraries of YAC DNA cloned into $\lambda$ phage or plasmids can be used to isolate vector-insert junctions. Although these techniques are reliable, they are too time consuming and labor intensive for analysis of large numbers of clones. We have found that total yeast DNA partially digested with $\mathrm{MboI}$ and size fractionated by gel electrophoresis is suitable for ligation to replacement vectors such as $\lambda$ DASHII (Stratagene) or EMBL3. We routinely screen $10^{4}-10^{5}$ plaques by hybridization with a ${ }^{32} \mathrm{P}$-labeled LA-specific (2.7-kb BamHI-PvuII pBR322 fragment) or RA-specific (1.7-kb BamHI-PvuII pBR322 fragment) probe to obtain several clones containing the pYAC4 vector-insert junction.

A scheme for directly subcloning into modified pUC19 plasmid vectors has been developed by Chaplin and Geraghty $^{(35,36)}$ (Fig. 2A). Total yeast DNA is double digested with a rare-cutting restriction enzyme that cleaves in the vector arm and a frequent-cutting restriction enzyme that cleaves in the genomic insert. The frequent-cutting restriction endonuclease may also cleave in the vector arm but not in the interval between the rare-cutting restriction site and the EcoRI cloning site. For LA vector-insert junctions, the DNA is digested with ClaI and either SacI, KpnI, SmaI, BamHI, XbaI, or SphI. For the RA, SalI is combined with either SacI, KpnI, SmaI, BamHI, XbaI, $S p h \mathrm{I}$, or HindIII. The use of several frequent-cutting restriction enzymes increases the chances of obtaining a clonable fragment. After digestion, an aliquot of DNA is subjected to gel electrophoresis, blotted, and hybridized with either LA or RA probes (Fig. 2B). Subsequently, vector-insert fragments in the range of $2-7 \mathrm{~kb}$ are size selected by gel electrophoresis. The purified DNA fragments are ligated to a modified pUC19 vector that was double digested with the appropriate restriction enzyme. The original pUC19 vector was modified by subcloning a 475-bp TaqI stuffer fragment (derived from positions 653-1128 of pBR322 ) into the Accl site of the polylinker. This creates the vectors pUC19-ES and HS (Fig. 2A). In pUC19-ES, the AccI site adjacent to the PstI site, but not that adjacent to the $\mathrm{XbaI}$ site, is preserved. Conversely, in pUC19-HS, the Accl site adjacent to the $\mathrm{Xbal}$ fragment is preserved, whereas that adjacent to the PstI site is destroyed. The AccI site is compatible with ClaI-digested DNA. The presence of the stuffer fragment after digestion and gel electrophoresis confirms that the vector has been prepared appro- priately. After ligating the size-selected restriction fragments to the vector, $\mathrm{Rec}^{-}$ E. coli are transformed and plated at a density of $\sim 200$ colonies per plate. Colony lifts are hybridized with either ${ }^{32} \mathrm{P}$ labeled LA or RA probes (Fig. 2B). These probes can be prepared by PCR or by restriction endonuclease digestion and random priming of the isolated fragments. These pBR322 sequences are adjacent to the SUP4 region of pYAC4 and do not hybridize to pUC19. Approximately $1-4 \%$ of the colonies should hy-

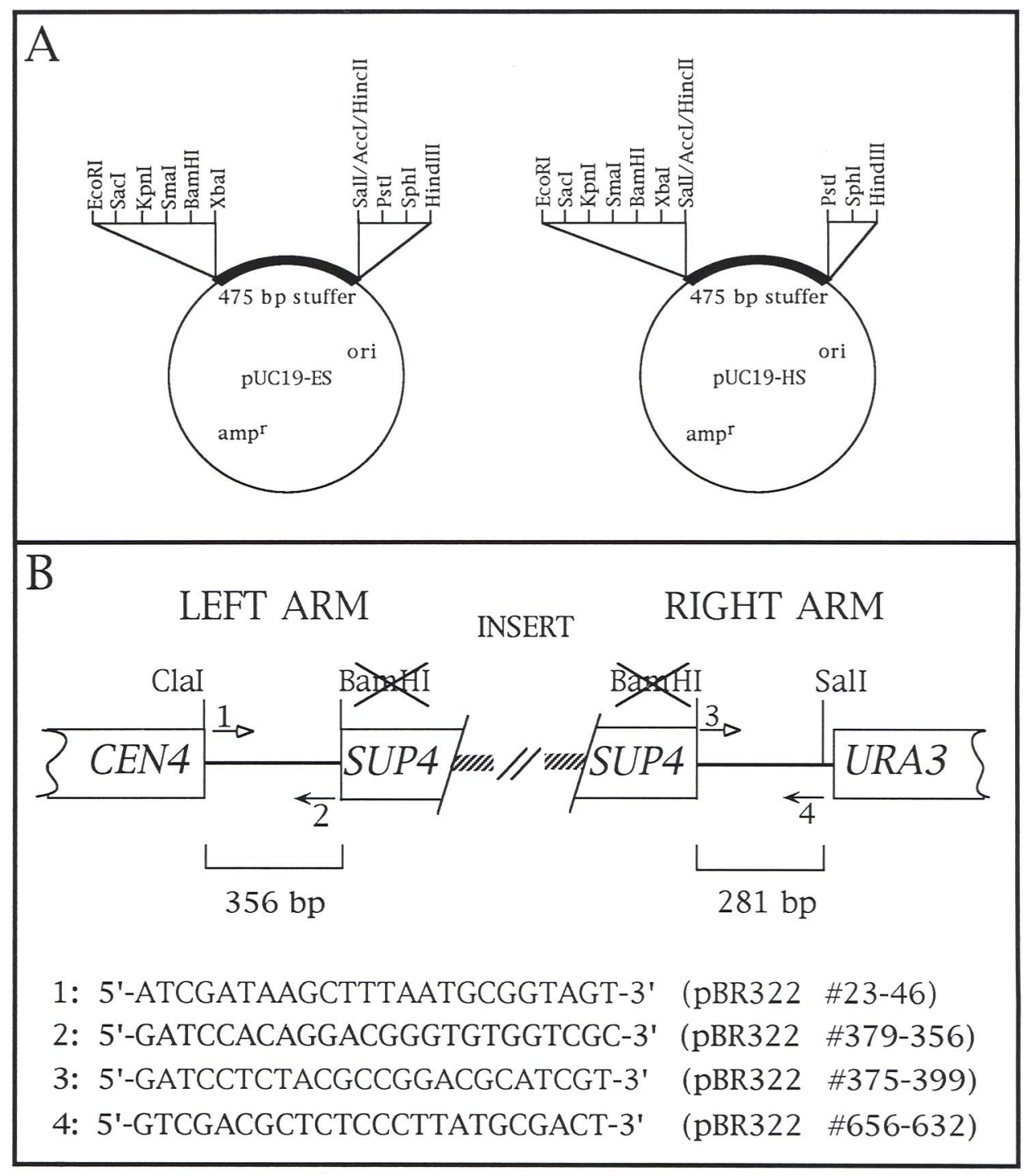

FIGURE $2(A)$ Modified pUC19 cloning vectors for isolating YAC vector-insert junctions. pUC19 vectors were modified by inserting, in both orientations, a 475-bp TaqI stuffer fragment. Appropriate double digestion of the vector is confirmed by gel electrophoresis and visualization of the stuffer fragment. $(B)$ Reagents for plasmid cloning of YAC end fragments. pYAC vector arm probe. Map of pYAC vector demonstrating positions and sequences of left- and right-arm-specific probes and primers. The location of the pBR322 BamHI sites that were destroyed during vector construction are shown for orientation. Adapted, with permission, from Chaplin. ${ }^{(36)}$ These probes will not hybridize to the pUC vectors. 
bridize with the probes. The high percentage of positive clones is caused by enrichment of vector-insert fragments afforded by double digestion with rareand frequent-cutting restriction enzymes and subsequent size fractionation. Using this technique, Bronson et al. ${ }^{(35)}$ constructed a YAC contig spanning a portion of the human major histocompatibility complex that contains the class I genes HLA-B and HLA-C.

\section{GENOMIC SEQUENCING}

Described by Coulson et al., ${ }^{(37)}$ this genomic sequencing technique employs the use of a vector-arm-specific primer and yeast miniprep DNA in a thermocycling dideoxy-sequencing reaction. Because this technique does not employ endonuclease digestion, ligation, or subcloning steps, it is a direct method for obtaining sequence information from YAC vector-insert junctions.
Primers specific for portions of SUP4 gene inclusive of the EcoRI cloning site are synthesized for both the LA (pYACL2) and the RA (pYACR4) (Fig. 3). These primers are end-labeled in a kinasing reaction with $\left[\gamma^{32} \mathrm{P}\right] \mathrm{dATP}$ and then used directly in a 10-cycle (denaturation at $95^{\circ} \mathrm{C}$ for $1 \mathrm{~min}$, annealing at $50^{\circ} \mathrm{C}$ for 2 min, elongation at $72^{\circ} \mathrm{C}$ for $2 \mathrm{~min}$, dideoxy-sequencing reaction containing $1-2.5 \mu \mathrm{g}$ of genomic DNA. The amplification products are separated by electrophoresis through $6 \%$ denaturing acrylamide gels. Gels are dried and exposed to $\mathrm{X}$-ray film that is backed with intensifying screens. After a 3-day exposure, $\sim 150$ bp of sequence can be read. Analysis of 180 YACs containing $C$. elegans DNA provided sequence from 275 ends. No sequence and double sequence ladders were obtained from $<10 \%$ and $<3 \%$, respectively, of the reactions. These problems were secondary to inadequate minipreps and multiple transformants,
respectively.PCR primer pairs that amplified $>100$ bp fragments were developed successfully from $\sim 250$ of the DNA sequences. The failure of primers to amplify the correct fragment was caused by misreading of inadequate genomic sequence, misreading of good sequence, and synthesis of self-complementary oligonucleotides. Us-ing the PCR fragments to probe DNA filters, 40 linkages were detected between the $\sim 200$ contigs spanning the C. elegans genome.

\section{PCR-BASED METHODS}

Alu-vector PCR

Approximately one million copies of the Alu repeat sequence are interspersed in the human genome. ${ }^{(38)}$ Although they are present with an average spacing of 4 $\mathrm{kb},{ }^{(39)}$ Alu elements tend to cluster in GC-rich, R bands. ${ }^{(40)}$ The 300-bp Alu element consists of a head-to-tail dimer of

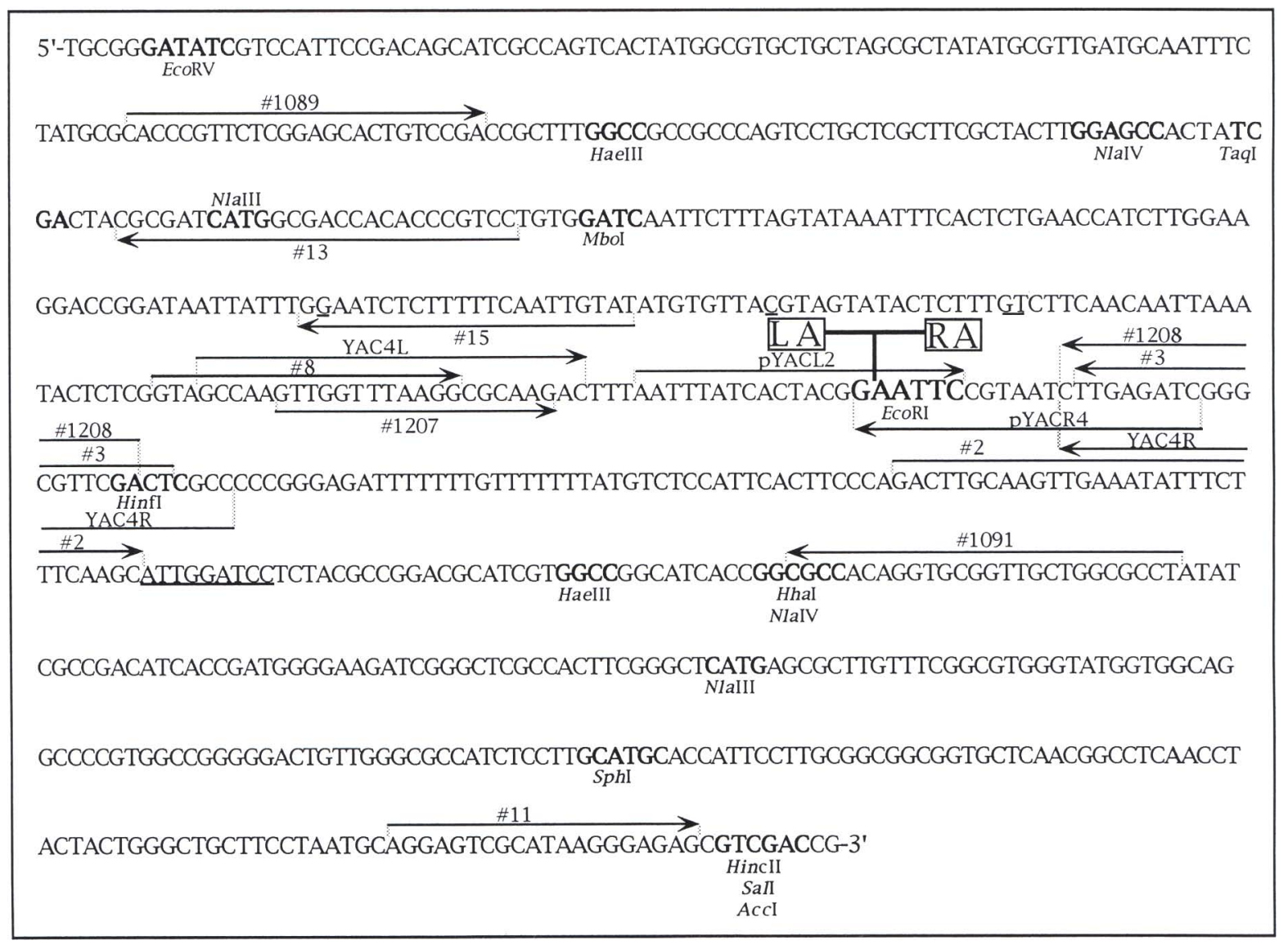

FIGURE 3 DNA sequence flanking the pYAC4 EcoRI cloning site. The location of various left arm (LA) and right arm (RA) vector primers used for different PCR applications (see text) are depicted by arrows. The vertical line demarcates LA from RA. Insertions and point mutations not predicted by the composite sequence (i.e., pBR322 and SUP4) are underlined. This sequence was obtained by standard dideoxy chain termination methods (G.A. Silverman, unpubl.). 
two $~ 130$-bp segments that share $>70 \%$ homology with an original sequence. ${ }^{(38)}$ The $3^{\prime}$ end of each Alu monomer is AT rich, while the remaining portion is $62 \%$ GC rich. ${ }^{(38)}$ In addition, there is a $31-b p$ insertion in the right-half monomer that is unique to primates. ${ }^{(38)}$ Although different $A l u$ families have evolved, ${ }^{(41)}$ Nelson et al. ${ }^{(42)}$ developed primers, which permit inter-Alu amplification, from a consensus sequence $^{(43)}$ (Fig. 4A). Primers that direct DNA synthesis either $5^{\prime}$ (\#278) or 3' (TC65) of the Alu sequence are capable of generating specific products from somatic-cell hybrids, YACs, or $\lambda$ phage containing human $\mathrm{DNA}^{\left({ }^{(42)}\right.}$ Subsequently, Nelson et al. ${ }^{(44)}$ extended their observations by demonstrating that YAC vector-insert junctions could be amplified by using either of the Alu primers in combination with either a LA (YAC4L) or RA (YAC4R) YAC vector primer (Figs. 3 and 4B). Products were detected using 100-500 ng of total yeast DNA and relatively routine PCR conditions (100 $\mu$ l volume: $1 \mu \mathrm{M}$ each primer,
$50 \mathrm{~mm} \mathrm{KCl}, 10 \mathrm{~mm}$ Tris-HCl, $1.5 \mathrm{~mm}$ $\mathrm{MgCl}_{2}, 300 \mu \mathrm{M}$ each dNTP, 2.5 units of Taq polymerase, and 35 cycles at $94^{\circ} \mathrm{C}$ for $1 \mathrm{~min}, 55^{\circ} \mathrm{C}$ for $45 \mathrm{sec}, 68^{\circ} \mathrm{C}$ for 5 $\mathrm{min})$. DNA sequence analysis of one PCR fragment confirmed that it was a vectorinsert junction. Amplification products from both ends were obtained from $60 \%$ of the YAC clones. The lower success rate of Alu-vector PCR relative to the other PCR-based methods (see below) is likely caused by the length of the DNA segment between the Alu element and the vector arm being beyond the range that can be amplified by current PCR methods. Nonetheless, the simplicity and versatility of this technique (i.e., no endonuclease digestion or ligation steps) makes this method an indispensable ad junct to most large-scale walking projects. Indeed, 8.0-, 2.2-, and 1.2-Mb YAC contigs encompassing $\mathrm{Xq} 26,{ }^{(45)}$ the Huntington's disease gene, ${ }^{(12)}$ and the iduronate sulfatase gene, ${ }^{(46)}$ respectively, have been constructed, in part, using Alu-vector PCR.
The generation of $A l u$-vector PCR products may be enhanced by using different sets of Alu primers. Breukel et al. ${ }^{(47)}$ detected PCR products in 14 of 21 YACs using different nondegenerate (Alu3 or Alu5) or degenerate primers (PDJ33 or PDJ34) (Fig. 4A).

\section{Anchor-vector PCR}

Anchor, oligo-cassette, or ligation-mediated PCR methods can be used to isolate an unknown DNA fragment that is adjacent to a known sequence. This is achieved by ligating a known primer template to the unknown sequence. The presence of known flanking templates permits the intervening sequence to be amplified in a PCR assay. Riley et al. ${ }^{(48)}$ adapted this technique to isolate vectorinsert junctions from YAC clones. YAC DNA is digested with a restriction enzyme that cleaves at a known site in the vector arm and at an unknown location in the genomic insert. Restriction enzymes that cleave between the vector-

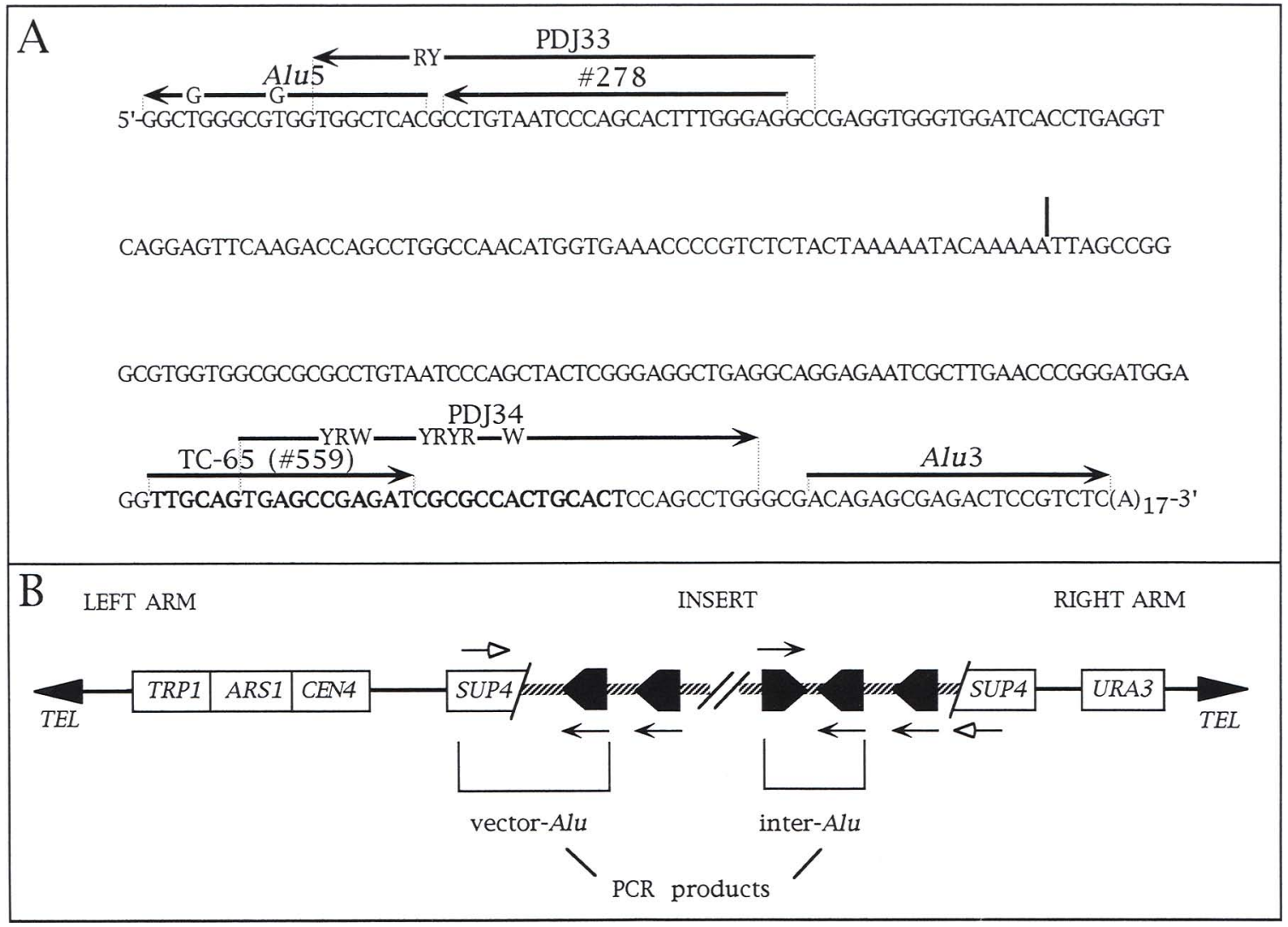

FIGURE 4 Alu-vector PCR. (A) Consensus human Alu sequence. The location of Alu primers for different PCR applications (see text) are depicted by arrows. The vertical line demarcates the left from the right Alu monomer. The 31-bp sequence unique to primates is in boldface type. Abbreviations: $\mathrm{Y}=\mathrm{C}$ or $\mathrm{T}, \mathrm{R}=\mathrm{G}$ or $\mathrm{A}, \mathrm{W}=\mathrm{T}$ or $\mathrm{A}$. $(B)$ Example of $A l u$-vector vs. inter-Alu PCR. Adapted, with permission, from Nelson et al. ${ }^{(42)}$ 


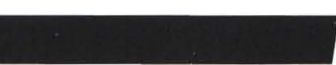

by Cold Spring Harbor Laboratory Press

primer template sequence and the EcoRI cloning site must be avoided. For the isolation of LA fragments, the restriction enzymes AluI, BglII, EcoRV, HincII, Hinfl, PvuII, and RsaI can be used. For the RA, AccI, BglII, EcoRV, HincII, RsaI, and PvuII can be used. Additional enzymes can be used if different vector-arm primers are selected (Fig. 3). The use of different restriction enzymes increases the chances of obtaining a vector-insert fragment that can be amplified by the PCR. After digestion, the DNA is ligated to a partial duplex DNA molecule or vectorette to form a library.

The vectorette consists of two oligonucleotides, a universal bottom strand that serves as an anchor template and a partially complementary top strand that serves as a linker (Fig. 5A). A similar type of cassette has been developed by Roux and Dhanarajan ${ }^{(49)}$ (Fig. 5B). The vectorette oligonucleotides contain 11 or 12 bp of complementary sequence that flank $29 \mathrm{bp}$ of noncomplementary sequence. By adding or deleting bases to the 5 ' end of the upper strand, vectorettes compatible with blunt- or sticky-ended genomic fragments can be constructed. This is a potential drawback because the cost of synthesizing an array of several upper strand oligonucleotides may be prohibitive. In practice, however, the blunt-end vectorette is satisfactory for many applications as it is compatible for ligation with DNA fragments digested with either RsaI, HinclI, PvuII, or EcoRV.

The vector-insert junctions are amplified from the vectorette library using LA (\#1089) or RA (\#1091) vector primers (Fig. 3) and the universal vectorette primer (\#224, Fig. 5A). The universal vectorette primer DNA sequence is identical to that of the "noncomplementary" region of the anchor template (bottom strand, Fig. 5A). The noncomplementary region of the vectorette con fers selective amplification to this scheme. Because there is no template available for the universal vectorette during the first round of DNA synthesis, only YAC vector-insert-vectorette fragments will be amplified (Fig. 6). During this first round, YAC vector primers synthesize a new top strand that is complementary to the bottom strand. This generates a template for universal vectorette primer and permits subsequent logarithmic amplification of the vector-insert-vectorette fragment (Fig. 6). Because no first strand synthesis occurred, other DNA frag-

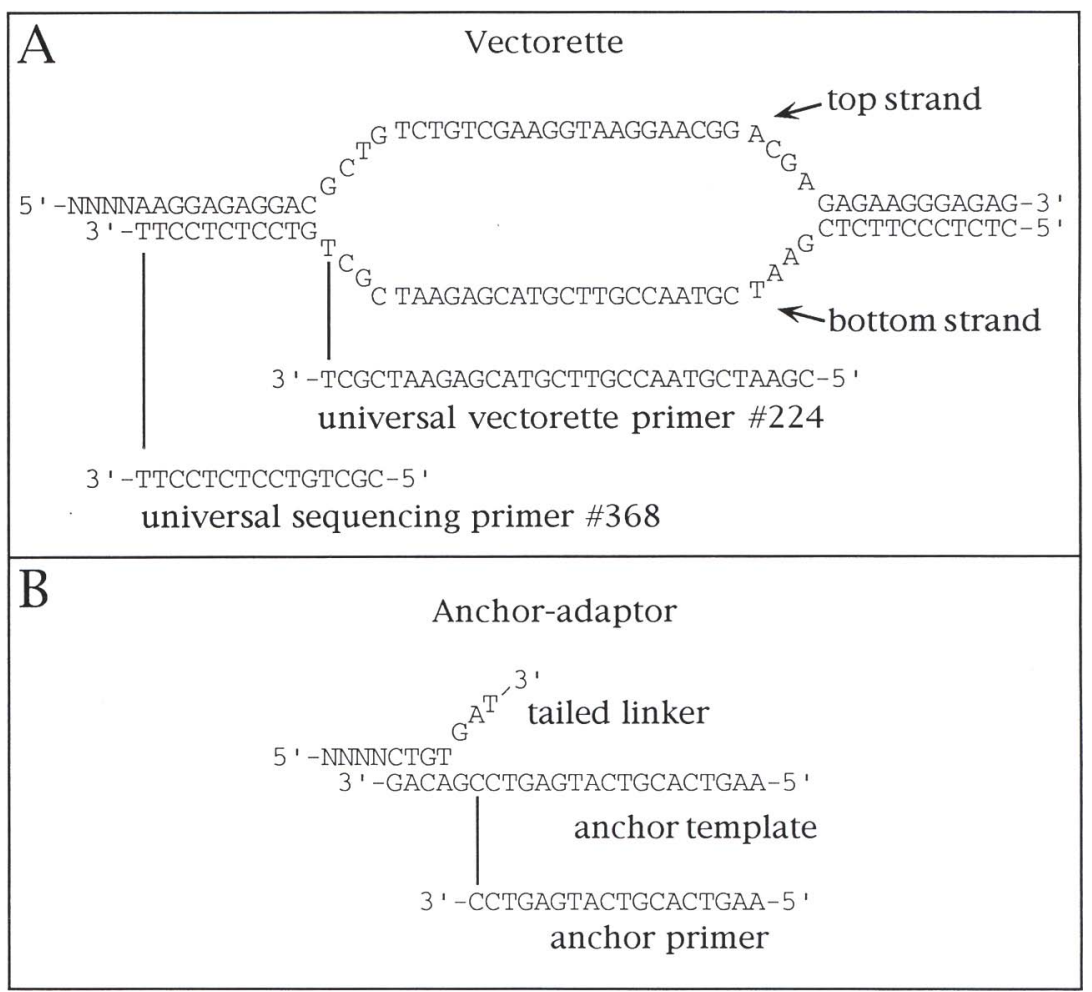

FIGURE 5 Anchors for ligation-mediated PCR. $(A)$ Vectorette or bubble cassette. Adapted, with permission, from Riley et al. ${ }^{(48)}(B)$ Anchor-adapter. Adapted, with permission, from Roux and Dhanarajan. ${ }^{(49)}$

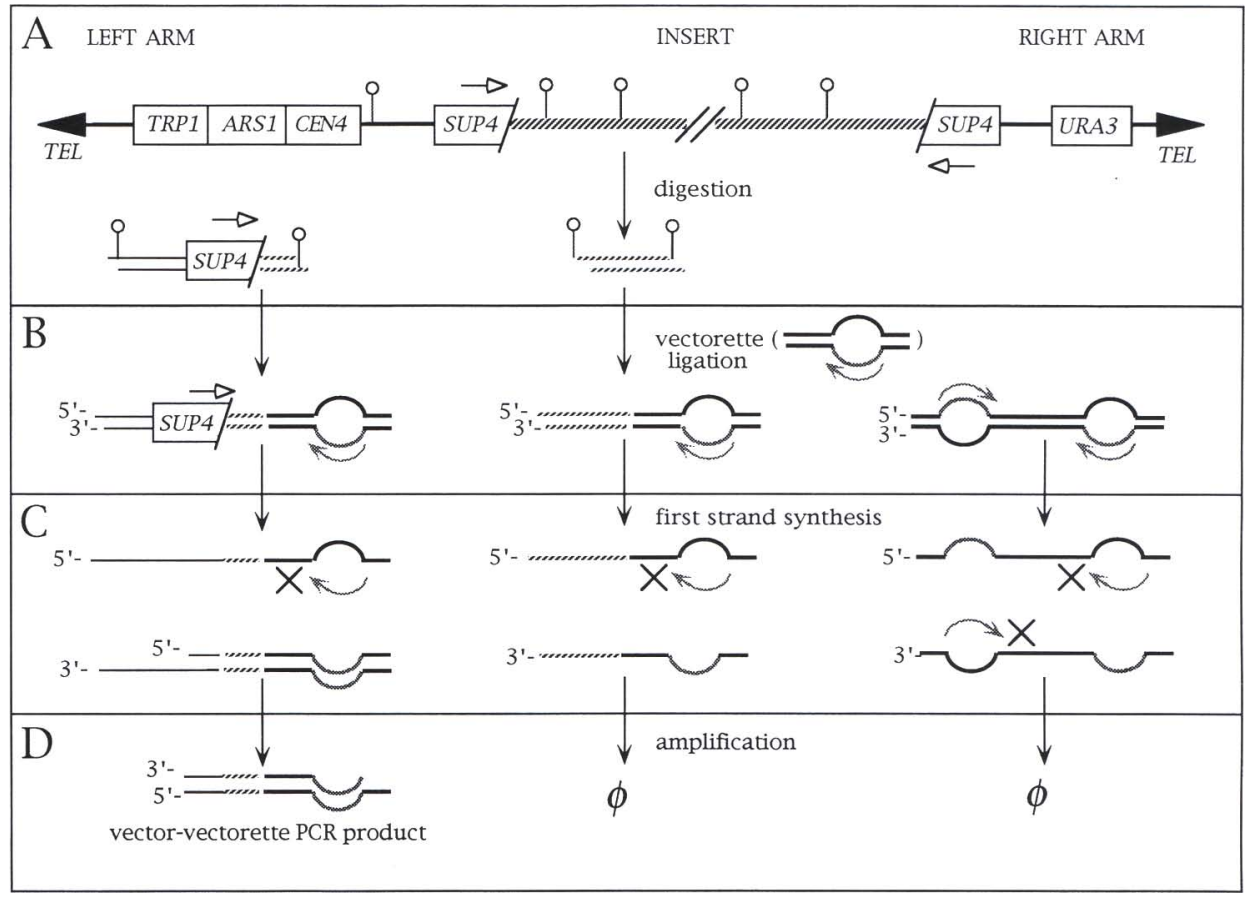

FIGURE 6 Vectorette-YAC vector PCR. $(A)$ YAC DNA is digested with a restriction endonuclease to yield a vector-insert fragment. $(B)$ Vectorettes are ligated to restriction fragments to yield vectorette libraries. $(C)$ Vectorette libraries are amplified using vector (open arrow in $B$ ) and universal vectorette (stippled arrow) primers. First-strand synthesis occurs only with the vector primer, as there is no template for the universal vectorette primer. $(D)$ Only vectorette-vector fragments, extended by the vector primer, are available for logarithmic amplification with the universal vectorette primer. 
ments ligated to the vectorette will not be amplified by the vectorette primers. PCR products generated by this technique can be reamplified or sequenced using a nested YAC vector (LA \#1207 or RA \#1208, Fig. 3) or vectorette primers (\#368, Fig. 5A). DNA sequence analysis of amplified fragments confirmed that they contained vector-insert junctions. ${ }^{(48)}$

The YAC vector-vectorette amplification technique has proved to be a robust method for isolating vector-insert junctions. Success rates exceed $90 \%$. The construction of 2.6- and 1.5-Mb YAC contigs spanning the human dystrophin gene ${ }^{(50)}$ and the region between D21S13 and $\mathrm{D} 21 \mathrm{~S} 16,{ }^{(51)}$ respectively, attest to the utility of this technique.

Other anchor-vector PCR methods have been developed to isolate vectorinsert junctions from YAC clones. Kere et al. ${ }^{(52)}$ adapted the method of Mueller and Wold ${ }^{(53)}$ to isolate 14 insert ends from YACs that map to the human $X$ chromosome. Success rates ranged from 80 to $85 \%$. Capture PCR (CPCR) was developed by Langerstrom et al. ${ }^{(54)}$ to facilitate end-fragment isolation using genomic DNA or yeast colonies. DNA is digested and ligated to a linker cassette. The ligation products are linearly amplified using a 5' biotinylated YAC vector arm primer. These extension products, now flanked with known template sequences, are purified by capture to streptavidin-coated beads or supports. After extensive washing, the extension products are logarithmically amplified using YAC vector and linker primers. Using only two restriction enzymes, AluI and $R s a \mathrm{I}$, the success rate was $\sim 85 \%$.

\section{Inverse PCR}

Inverse PCR was developed also to isolate an unknown DNA sequence adjacent to a known sequence. ${ }^{(55-57)}$ Subsequently, this technique was modified and adapted for isolation of pYAC4 vector-insert junctions. ${ }^{(58,59)}$ First, sets of oligonucleotide primers in inverse orientation (i.e., $3^{\prime} \leftarrow 5^{\prime} / 5^{\prime} \rightarrow 3^{\prime}$ ) are synthesized for both the LA and RA (Fig. 3). Next, YAC DNA is digested with a restriction enzyme that cleaves in the vector arm and at an unknown location within the vector insert. MboI, NlaIII, TaqI, NlaIV, HaeIII, EcoRV, and RsaI can be used for the LA; and NlaIV, HaeIII, NlaIII, $H h a \mathrm{I}, S p h \mathrm{I}, A c c \mathrm{I}$, and HincII for the RA (Fig. 3). The use of multiple restriction enzymes enhances the probability of isolating a useful fragment. However, it may be useful to start with those enzymes that can be used on both vector arms. As with vectorette PCR, care must be taken to avoid the use of restriction endonucleases that cleave in the region between the two primer templates and the EcoRI cloning site. After digestion, the DNA is diluted to a concentration between 0.2 and $2.0 \mu \mathrm{g} / \mathrm{ml}$. Ligation of DNA under these conditions favors the formation of monomer circles (Fig. 7). Circularization reorients the vector primers and permits amplification of the intervening genomic insert (Fig. 7). Confirmation of the appropriate terminal fragment can be verified by DNA sequencing or hybridization of the labeled fragment to blots of YAC DNA. ${ }^{(58,59)}$

In comparison with the other PCRbased techniques used to isolate vectorinsert junctions, this technique may be more difficult to perfect. However, once the technique is established, individual laboratories report success rates that rival or exceed those of the ligation-mediated methods. We have isolated YAC vector-insert junctions in 26 of 26 attempts. The fragments range in size from 26 to 1250 bp with a mean of $\sim 500$ bp. Al- though a single set of PCR primers can be used for each vector arm, we have found that the combination of certain vector arm primers and restriction enzymes are more successful in yielding PCR fragments. For the LA, we use primer combinations $\# 5$ and \#8 with DNA digested with $\mathrm{MboI}$ and NlaIII (Fig. 3 ). Primers $\# 13$ and $\# 8$ are used with TaqI-, NlaIV-, HaelII-, and EcoRV-digested DNA. For the RA, primers \#2 and \#3 are used with NlaIV, HaeIII, NlaIII, HhaI, and SphI digests; and primers \#11 and \#3 with $A c c \mathrm{I}$ and HincII digests.

Megabase-size YAC contigs spanning the $\mathrm{BCL} 2,^{(59)}$ Huntington's disease, ${ }^{(11)}$ and $\mathrm{APC}^{(9,10)}$ genes have been constructed using inverse PCR. In addition, Gill and Somerville used a single restriction enzyme and inverse PCR to isolate 12 of 14 end fragments from a modified pYAC vector containing Arabidopsis thaliana DNA. ${ }^{(60)}$ Collectively, these studies demonstrate that inverse PCR is an efficient method for isolating large numbers of vector-insert junctions.

\section{CONCLUSIONS}

Methods to isolate DNA fragments or sequences from the ends of the genomic

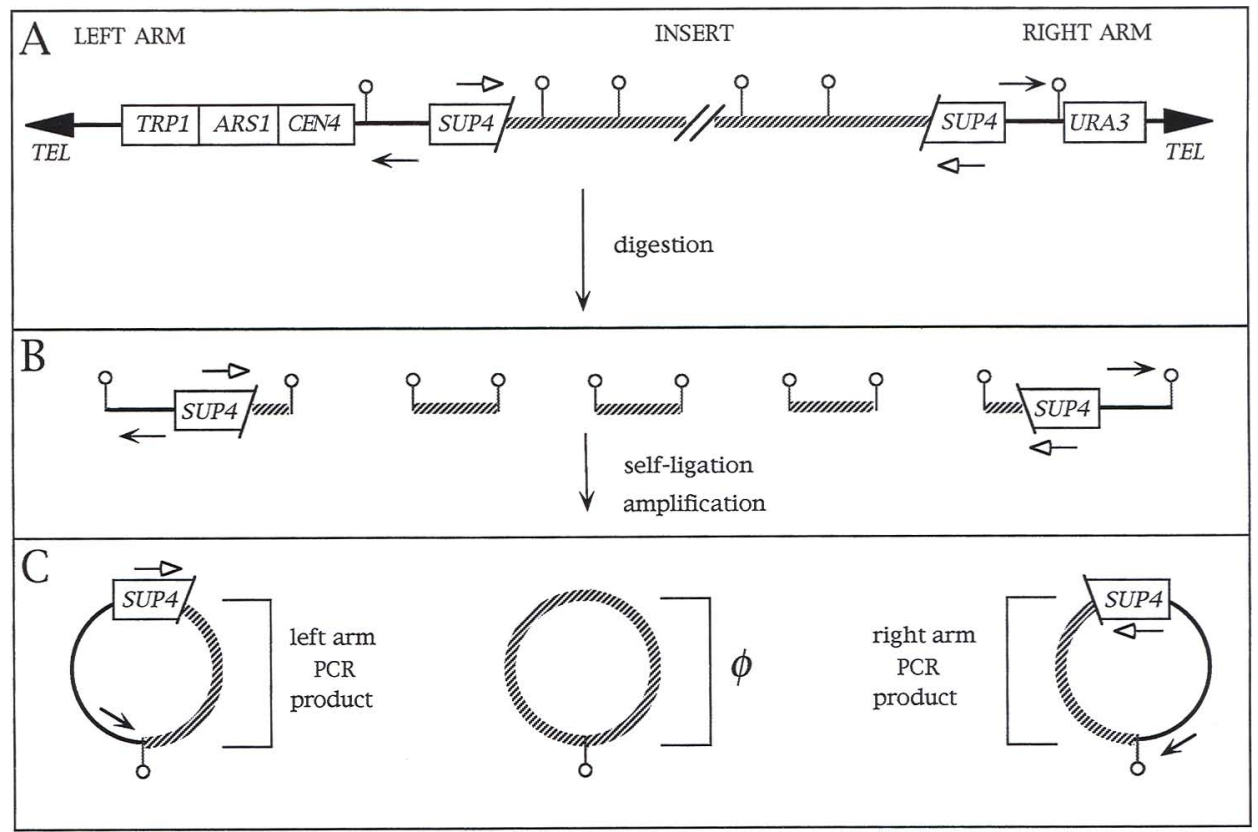

FIGURE 7 Inverse PCR $(A)$ YAC DNA is digested with a restriction enzyme that cleaves at a known location in the vector arm and at an unknown location in the genomic insert $(O)$. Templates for vector primers are oriented inversely and would direct DNA synthesis in opposite direction (arrows). (B) Restriction fragments are self-ligated to form monomer circles. (C) Circularized vector-insert junctions have reoriented primer templates that permit amplification of intervening genomic insert. 
inserts are important adjuncts to YAC cloning. End fragments generated by these techniques facilitate positional cloning of disease-related genes, the closure of gaps between sets of clones, and the integration of contigs into high-resolution physical maps. Although numerous techniques have been developed to isolate end fragments with a high rate of success, no superior technique has emerged. In part, this is attributable to the task at hand. For example, if the goal is to isolate a relatively large DNA fragment for FISH studies, then plasmid rescue or conventional $\lambda$ phage cloning, rather than PCR, is more likely to yield an end fragment of the appropriate size. In contrast, if the goal is to generate numerous STSs to complete the assembly of a contig spanning an entire mammalian chromosome, then PCR-based techniques may be more advantageous because of their high throughput.

The practical considerations of time and expense must be considered when developing strategies to isolate end fragments. Plasmid rescue and conventional cloning of end fragments can be initiated with reagents readily available to most molecular biology laboratories. However, these techniques may require weeks to isolate the desired fragment. Although time may not be a major consideration when isolating a few end fragments, it becomes a determining factor when large numbers of clones are involved. Using PCR-based techniques, an STS can be developed from the end of a YAC clone insert within a few days. However, when using PCR-based techniques, the cost of oligonucleotides and thermostable polymerases should be taken into account. Use of automation for the isolation of large numbers of end fragments can also help in reducing effort and costs expended on each STS.

\section{ACKNOWLEDGMENTS}

This work was supported by grants from the National Institutes of Health (HD28475), the Hearst Fund, and the March of Dimes (0859). I thank Gail Bruns and Sandra Schneider for critical comments, and Kelly Ames for preparation of the manuscript.

\section{REFERENCES}

1. Schlessinger, D. 1990. Yeast artificial chromosomes: tools for mapping and analysis of complex genomes. Trends Genet. 6: 248-258.

2. Hieter, P., C. Connelly, J. Shero, M.K. McCormick, S. Antonarakis, W. Pavan, and R. Reeves. 1990. Yeast artificial chromosomes: Promises kept and pending. In $\mathrm{Ge}$ nome analysis: Genetic and physical mapping (ed. K.E. Davies and S.M. Tilghman), vol. 1, pp. 83-120. Cold Spring Harbor Laboratory Press, Cold Spring Harbor, New York.

3. Burke, D.T. 1991. The role of yeast artificial chromosome clones in generating genome maps. Curr. Opin. Genet. Dev. 1: 69 74.

4. Foote, S., D. Vollrath, A. Hilton, and D.C. Page. 1992. The human Y chromosome: Overlapping DNA clones spanning the euchromatic region. Science 258: 60-66.

5. Chumakov, I., P. Rigault, S. Guillou, P. Ougen, A. Billaut, G. Guasconi, P. Gervy, I. LeGall, P. Soularue, L. Grinas, L. Bougueleret, C. Bellanne-Chantelot, B. Lacroix, E. Barillot, P. Gesnouin, S. Pook, G. Vaysseix, G. Frelat, A. Schmitz, J.-L. Sambucy, A. Bosch, X. Estivill, J. Weissenbach, A. Vignal, H. Riethman, D. Cox, D. Patterson, K. Gardiner, M. Hattori, Y. Sakaki, H. Ichikawa, M. Ohki, D. Le Paslier, R. Heilig, S. Antonarakis, and D. Cohen. 1992. Continuum of overlapping clones spanning the entire human chromosome $21 \mathrm{q}$ (see comments). Nature 359: 380-387.

6. Coulson, A., R. Waterston, J. Kiff, J. Sulston, and Y. Kohara. 1988. Genome linking with yeast artificial chromosomes. Nature 335: 184-186.

7. Hartl, D.L., J.W. Ajioka, H. Cai, A.R. Lohe, E.R. Lozovskaya, D.A. Smoller, and I.W Duncan. 1992. Towards a Drosophila genome map. Trends Genet. 8: 70-75.

8. Marchuk, D.A., R. Tavakkol, M.R. Wallace, B.H. Brownstein, P. Taillon-Miller, C.T. Fong, E. Legius, L.B. Andersen, T.W. Glover, and F.S. Collins. 1992. A yeast artificial chromosome contig encompassing the type 1 neurofibromatosis gene. Genomics 13: 672-680.

9. Groden, J., A. Thliveris, W. Samowitz, M Carlson, L. Gelbert, H. Albertsen, G. Joslyn, J. Stevens, L. Spirio, M. Robertson, L. Sargeant, K. Krapcho, E. Wolff, R. Burt, J.P. Hughes, J. Warrington, J. McPherson, J. Wasmuth, D. Le Paslier, H. Abderrahim, D. Cohen, M. Leppert, and R. White. 1991. Identification and characterization of the familial adenomatous polyposis coli gene. Cell 66: 589-600.

10. Joslyn, G., M. Carlson, A. Thliveris, H. Albertsen, L. Gelbert, W. Samowitz, J. Groden, J. Stevens, L. Spirio, M. Robertson, L. Sargeant, K. Krapcho, E. Wolff, R. Burt, J.P. Hughes, J. Warrington, J. McPherson, J. Wasmuth, D. Le Paslier, H. Abderrahim, D. Cohen, M. Leppert, and R. White. 1991. Identification of deletion muta- tions and three new genes at the familial polyposis locus. Cell 66: 601-613.

11. Zuo, J., C. Robbins, M.P. Taillon, D.R. Cox, and R.M. Myers. 1992. Cloning of the Huntington disease region in yeast artificial chromosomes. Hum. Mol. Genet. 1: 149-159.

12. Bates, G.P., J. Valdes, H. Hummerich, S. Baxendale, D.L. Le Paslier, A.P. Monaco, D. Tagle, M.E. MacDonald, M. Altherr, M. Ross, B.H. Brownstein, D. Bentley, J.J. Wasmuth, J.F. Gusella, D. Cohen, F. Collins, and H. Lehrach. 1992. Characterization of a yeast artificial chromosome contig spanning the Huntington's disease gene candidate region. Nature Genet. 1: 180-187.

13. Olson, M.V., J.E. Dutchik, M.Y. Graham, G.M. Brodeur, C. Helms, M. Frank, M. MacCollin, R. Scheinman, and T. Frank. 1986. Random-clone strategy for genomic restriction mapping in yeast. Proc. Natl. Acad. Sci. 83: 7826-7830.

14. Kohara, Y., K. Akiyama, and K. Isono. 1987. The physical map of the whole $\mathrm{E}$. coli chromosome: application of a new strategy for rapid analysis and sorting of a large genomic library. Cell 50: 495-508.

15. Coulson, A., J. Sulston, S. Brenner, and J. Karn. 1986. Toward a physical map of the genome of the nematode Caenorhabditis elegans. Proc. Natl. Acad. Sci. 83: 78217825.

16. Riles, L., J.E. Dutchik, A. Baktha, B.K. McCauley, E.C. Thayer, M.P. Leckie, V.V. Braden, J.E. Depke, and M.V. Olson. 1993. Physical maps of the six smallest chromosomes of Saccharomyces cerevisiae at a resolution of 2.6 kilobase pairs. Genetics 134: 81-150.

17. Bellanne-Chantelot, C., E. Barillot, B. Lacroix, D. Le Paslier, and D. Cohen. 1991. A test case for physical mapping of human genome by repetitive sequence fingerprints: construction of a physical map of a $420 \mathrm{~kb}$ YAC subcloned into cosmids. $\mathrm{Nu}$ cleic Acids Res. 19: 505-510.

18. Bellanne-Chantelot, C., B. Lacroix, P. Ougen, A. Billault, S. Beaufils, S. Bertrand, I. Georges, F. Glibert, I. Gros, G. Lucotte, L. Susini, J.-J. Codani, P. Gesnouin, S. Pook, G. Vaysseix, J. Lu-Kuo, T. Ried, D. Ward, I. Chumakov, D. Le Paslier, E. Barillot, and D. Cohen. 1992. Mapping the whole human genome by fingerprinting yeast artificial chromosomes. Cell 70: 1059-1068.

19. Green, E.D., and M.V. Olson. 1990. Chromosomal region of the cystic fibrosis gene in yeast artificial chromosomes: a model for human genome mapping. Science 250: 94-98.

20. Olson, M., L. Hood, C. Cantor, and D. Botstein. 1989. A common language for physical mapping of the human genome (see comments). Science 245: 1434-1435. 21. Barillot, E., J. Dausset, and D. Cohen. 
1991. Theoretical analysis of a physical mapping strategy using random singlecopy landmarks. Proc. Natl. Acad. Sci. 88: 3917-3921.

22. Arratia, R., E.S. Lander, S. Tavare, and M.S. Waterman. 1991. Genomic mapping by anchoring random clones: a mathematical analysis. Genomics 11: 806-8027.

23. Ewens, W.J., C.J. Bell, P.J. Donnelly, P. Dunn, E. Matallana, and J.R. Ecker. 1991. Genome mapping with anchored clones: theoretical aspects. Genomics 11: 799805.

24. Palazzolo, M.J., S.A. Sawyer, C.H. Martin, D.A. Smoller, and D.L. Hartl. 1991. Optimized strategies for sequence-tagged-site selection in genome mapping. Proc. Natl. Acad. Sci. 88: 8034-8038.

25. Burke, D.T., G.F. Carle, and M.V. Olson. 1987. Cloning of large segments of exogenous DNA into yeast by means of artificial chromosome vectors. Science 236: 806-812.

26. Rose, M., P. Grisafi, and D. Botstein. 1984. Structure and function of the yeast URA3 gene: expression in Escherichia coli. Gene 29: 113-124.

27. Traver, C.N., S. Klapholz, R.W. Hyman, and R.W. Davis. 1989. Rapid screening of a human genomic library in yeast artificial chromosomes for single-copy sequences. Proc. Natl. Acad. Sci. 86: 58985902.

28. Shero, J.H., M.K. McCormick, S.E. Antonarakis, and P. Hieter. 1991. Yeast artificial chromosome vectors for efficient clone manipulation and mapping. Genomics 10: $505-508$.

29. Smith, D.R., A.P. Smyth, and D.T. Moir. 1990. Amplification of large artificial chromosomes. Proc. Natl. Acad. Sci. 87: 8242-8246.

30. Brownstein, B.H., G.A. Silverman, R.D. Little, D.T. Burke, S.J. Korsmeyer, D. Schlessinger, and M.V. Olson. 1989. Isolation of single-copy human genes from a library of yeast artificial chromosome clones. Science 244: 1348-1351.

31. Albertsen, H.M., H. Abderrahim, H.M. Cann, J. Dausset, D. Le Paslier, and D. Cohen. 1990. Construction and characterization of a yeast artificial chromosome library containing seven haploid human genome equivalents. Proc. Natl. Acad. Sci. 87: 4256-4260.

32. Anand, R., J.H. Riley, R. Butler, J.C. Smith, and A.F. Markham. 1990. A 3.5 genome equivalent multi access YAC library: Construction, characterisation, screening and storage. Nucleic Acids Res. 18: 1951-1956.

33. Larin, Z. and H. Lehrach. 1990. Yeast artificial chromosomes: an alternative approach to the molecular analysis of mouse developmental mutations. Genet. Res. 56: 203-208.

34. Hermanson, G.G., M.F. Hoekstra, D.L. McElligott, and G.A. Evans. 1991. Rescue of end fragments of yeast artificial chromosomes by homologous recombination in yeast. Nucleic Acids Res. 19: 4943-4948.

35. Bronson, S.K., J. Pei, M.P. Taillon, M.J. Chorney, D.E. Geraghty, and D.D. Chaplin. 1991. Isolation and characterization of yeast artificial chromosome clones linking the HLA-B and HLA-C loci. Proc. Natl. Acad. Sci. 88: 1676-1680.

36. Chaplin, D.D. and B.H. Brownstein. 1992. End fragment analysis using PCR amplification. In Current protocols in molecular biology (ed. F.M. Ausubel, R. Brent, R.E. Kingston, D.D. Moore, J.G. Seidman, J.A. Smith, and K. Struhl), pp. 6.10.6-6.10.19. Greene Publishing Associates, Inc./John Wiley \& Sons, Inc., New York.

37. Coulson, A., Y. Kozono, R. Shownkeen, and R. Waterston. 1991. The isolation of insert-terminal YAC fragments by genomic sequencing. Technique 3: 17-23.

38. Deininger, P.L., D.J. Jolly, C.M. Rubin, T. Friedmann, and C.W. Schmid. 1981. Base sequence studies of 300 nucleotide renatured repeated human DNA clones. J. Mol. Biol. 151: 17-33.

39. Hwu, H.R., J.W. Roberts, E.H. Davidson, and R.J. Britten. 1986. Insertion and/or deletion of many repeated DNA sequences in human and higher ape evolution. Proc. Natl. Acad. Sci. 83: 3875-3879.

40. Korenberg, J.R. and M.C. Rykowski. 1988. Human genome organization: Alu, lines, and the molecular structure of metaphase chromosome bands. Cell 53: 391-400.

41. Willard, C., H.T. Nguyen, and C.W. Schmid. 1987. Existence of at least three distinct Alu subfamilies. J. Mol. Evol. 26: 180-186.

42. Nelson, D.L., S.A. Ledbetter, L. Corbo, M.F. Victoria, R. Ramirez-Solis, T.D. Webster, D.H. Ledbetter, and C.T. Caskey. 1989. Alu polymerase chain reaction: A method for rapid isolation of human-specific sequences from complex DNA sources. Proc. Natl. Acad. Sci. 86: 66866690.

43. Kariya, Y., K. Kato, Y. Hayashizaki, S. Himeno, S. Tarui, and K. Matsubara. 1987. Revision of consensus sequence of human Alu repeats-A review. Gene 53: 1-10.

44. Nelson, D.L., A. Ballabio, M.F. Victoria, M. Pieretti, R.D. Bies, R.A. Gibbs, J.A. Maley, A.C. Chinault, T.D. Webster, and C.T. Caskey. 1991. Alu-primed polymerase chain reaction for regional assignment of 110 yeast artificial chromosome clones from the human X chromosome: Identification of clones associated with a disease locus. Proc. Natl. Acad. Sci. 88: 6157-6161.

45. Little, R.D., G. Pilia, S. Johnson, M. D'Urso, and D. Schlessinger. 1992. Yeast artificial chromosomes spanning 8 megabases and 10-15 centimorgans of human cytogenetic band Xq26. Proc. Natl. Acad. Sci. 89: 177-181.

46. Palmieri, G., V. Capra, G. Romano, M.
D'Urso, S. Johnson, D. Schlessinger, P. Morris, J. Hopwood, P. Di Natale, R. Gatti, and A. Ballabio. 1992. The iduronate sulfatase gene: isolation of a $1.2-\mathrm{Mb}$ YAC contig spanning the entire gene and identification of heterogeneous deletions in patients with Hunter syndrome. Genomics 12: $52-57$

47. Breukel, C., J. Wijnen, C. Tops, H. van der Klift, H. Dauwerse, and P.M. Khan. 1990. Vector-Alu PCR: a rapid step in mapping cosmids and YACs. Nucleic Acids Res. 18: 3097.

48. Riley, J., R. Butler, D. Ogilvie, R. Finniear, D. Jenner, S. Powell, R. Anand, J.C. Smith, and A.F. Markham. 1990. A novel, rapid method for the isolation of terminal sequences from yeast artificial chromosome (YAC) clones. Nucleic Acids Res. 18: 28872890.

49. Roux, K.H. and P. Dhanarajan. 1990. A strategy for single site PCR amplification of dsDNA: Priming digested cloned or genomic DNA from an anchor-modified restriction site and a short internal sequence. BioTechniques 8: 48-57.

50. Coffey, A.J., R.G. Roberts, E.D. Green, C.G. Cole, R. Butler, R. Anand, F. Giannelli, and D.R. Bentley. 1992. Construction of a $2.6-\mathrm{Mb}$ contig in yeast artificial chromosomes spanning the human dystrophin gene using an STS-based approach. Genomics 12: 474-484.

51. Butler, R., D.J. Ogilvie, P. Elvin, J.H. Riley, R.S. Finniear, G. Slynn, J.E. Morten, A.F. Markham, and R. Anand. 1992. Walking, cloning, and mapping with yeast artificial chromosomes: A contig encompassing D21S13 and D21S16. Genomics 12: 42-51.

52. Kere, J., R. Nagaraja, S. Mumm, A. Ciccodicola, M. D'Urso, and D. Schlessinger. 1992. Mapping human chromosomes by walking with sequence-tagged sites from end fragments of yeast artificial chromosome inserts. Genomics 14: 241-248.

53. Mueller, P.R. and B. Wold. 1989. In vivo footprinting of a muscle specific enhancer by ligation mediated PCR. (published erratum appears in 1990, Science 248: 802.) Science 246: 780-786.

54. Lagerstrom, M., J. Parik, H. Malmgren, J. Stewart, U. Pettersson, and U. Landegren. 1991. Capture PCR: efficient amplification of DNA fragments adjacent to a known sequence in human and YAC DNA. PCR Methods Applic. 1: 111-119.

55. Ochman, H., A.S. Gerber, and D.L. Hartl. 1988. Genetic applications of an inverse polymerase chain reaction. Genetics 120: 621-623.

56. Silver, J. and V. Keerikatte. 1989. Novel use of polymerase chain reaction to amplify cellular DNA adjacent to an integrated provirus (Published erratum appears in 1990, J. Virol. 64: 3150.) J. Virol. 63: 1924-1928.

57. Triglia, T., M.G. Peterson, and D.J. Kemp. 
1988. A procedure for in vitro amplification of DNA segments that lie outside the boundaries of known sequences. Nucleic Acids Res. 16: 8186.

58. Silverman, G.A., R.D. Ye, K.M. Pollock, J.E. Sadler, and S.J. Korsmeyer. 1989. Use of yeast artificial chromosome clones for mapping and walking within human chromosome segment 18q21.3. Proc. Natl. Acad. Sci. 86: 7485-7489.

59. Silverman, G.A., J.I. Jockel, P.H. Domer, R.M. Mohr, M.P. Taillon, and S.J. Korsmeyer. 1991. Yeast artificial chromosome cloning of a two-megabase-size contig within chromosomal band $18 \mathrm{q} 21$ establishes physical linkage between BCL2 and plasminogen activator inhibitor type-2. Genomics 9: 219-228.

60. Grill, E. and C. Somerville. 1991. Construction and characterization of a yeast artificial chromosome library of Arabidopsis which is suitable for chromosome walking. Mol. Gen. Genet. 226: 484-490.

Received September 13, 1993; accepted in revised form September 28, 1993. 


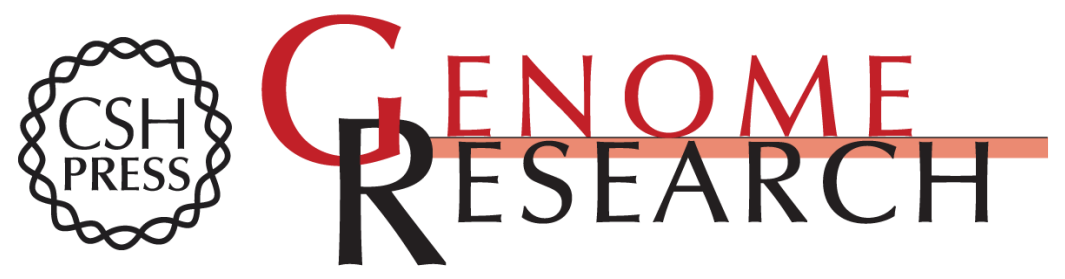

\title{
Isolating vector-insert junctions from yeast artificial chromosomes.
}

\author{
G A Silverman
}

Genome Res. 1993 3: 141-150

References This article cites 58 articles, 22 of which can be accessed free at:

http://genome.cshlp.org/content/3/3/141.full.html\#ref-list-1

\section{License}

Email Alerting Receive free email alerts when new articles cite this article - sign up in the box at the Service top right corner of the article or click here.

\section{Affordable, Accurate Sequencing.}

To subscribe to Genome Research go to: https://genome.cshlp.org/subscriptions 